\title{
Theranostics
}

Editorial

\section{Nanophotonics and Theranostics: Will Light Do the Magic?}

\author{
Dmitri O. Lapotko \\ Department of Biochemistry \& Cell Biology, Department of Physics \& Astronomy, Rice University, Houston, TX, USA \\ $\bowtie$ Corresponding author: dmitri.lapotko@rice.edu \\ (C) Ivyspring International Publisher. This is an open-access article distributed under the terms of the Creative Commons License (http://creativecommons.org/ \\ licenses/by-nc-nd/3.0/). Reproduction is permitted for personal, noncommercial use, provided that the article is in whole, unmodified, and properly cited.
}

Received: 2013.02.II; Accepted: 2013.02.15; Published: 2013.02.20

When speaking about optical approaches for theranostics, that is method that unites diagnosis, treatment and preferably treatment guidance in one procedure, it is good to recall what light already did in medicine as well as to consider the most pressing unresolved medical challenges that will benefit from new approaches including theranostics.

Light by itself is a universal form of energy (Figure 1) that can be rapidly delivered and converted into various medically-relevant forms such as heat, chemical and mechanical processes, sound and light, all of which are used for diagnosis and various therapies. The historic examples of light doing magic in medicine are the invention of the microscope in XVI century [1] and of "bloodless" laser surgery in XX century [2].
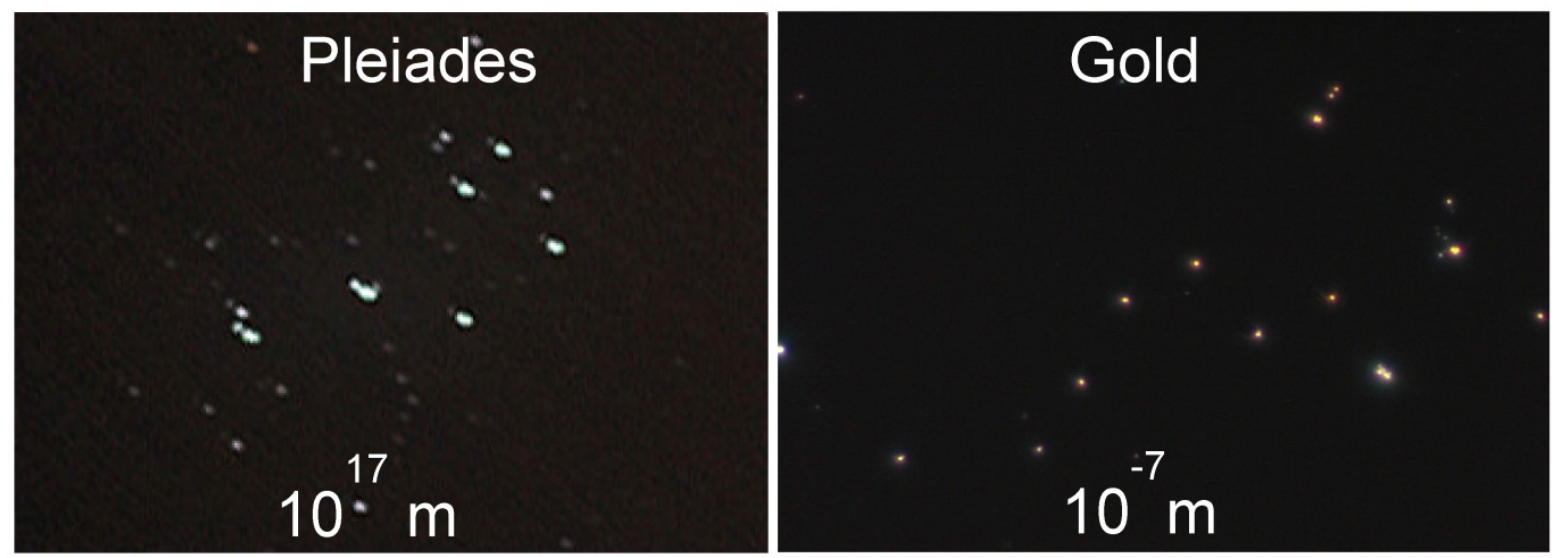

Present medical practices including surgery, and chemo- and radiation-therapies often suffer from an inability to remove or kill pathological cells when the latter are intertwined with functionally important organs and develop resistance to drugs and radiation. This forces clinicians to balance the risks of residual and recurrent disease with damaging important cells and organs, thus further complicating the disease by compromising the patient's quality of life and physiological health. The ultimate solution is to transform current macro-treatment into cell level theranostic tools which will rapidly and selectively detect and destroy only pathological cells while sparing nearby normal cells. Unfortunately, such a tool does not yet exist, and it is precisely here that the combination of light and nanotechnology is most promising.

Figure I. Range of light: from Mega - to nano-scale. 
The recently advances in chemistry allowed to employ a phenomenon of plasmon resonance [3] for the conversion of light into medically-relevant processes at nanoscale via metal (plasmonic) nanoparticles that show a million times higher optical absorbance than any natural molecules. Among them, gold nanoparticles have both the highest optical absorbance and the lowest toxicity. Interestingly, gold nanoparticles are not new. In fact, they were invented by Michael Faraday in the XIX century [4] and have been used in clinics for more than fifty years [5]. On the other hand, recent advances in laser technologies allowed the use of near-infrared continuous and pulsed light with the maximal tissue penetration depth (up to $10 \mathrm{~mm}$ [6]) and safety. Fiber optical technologies allowed the delivery of light even deeper, up to 300-400 $\mathrm{mm}$, thus enabling laser micro-surgery [7]. Finally, advances in conjugation chemistry made possible the precise delivery of gold nanoparticles to specific pathological cells, where these nanoparticles can be remotely and instantaneously activated with visible or near-infrared laser light. All the above created a unique interdisciplinary platform for the development and clinical translation of bio-nano-photonics. The current issue presents several research and review papers that report both the fundamentals and applications of light-activated nanoparticles in biology and medicine.

Diagnostic methods employ optical scattering [8-11], luminescence [11, 12] and fluorescence [9, 10] by nanoparticles and optical scattering and acoustic signals by plasmonic nanobubbles [13]. Cell-specific targeting and the internalization of nanoparticles provided high sensitivity and specificity of the diagnosis. Optical detection methods are mostly appropriate for in vitro and extra-corporeal applications with unlimited optical transparency. Photoacoustic methods [14] are better suited to in vivo applications, with poor optical transparency of opaque tissues. It is worth mentioning one specific animal model, zebrafish [15], that combines the physiology of a vertebrate animal with the high optical transparency of in vitro samples.

The therapeutic methods discussed in this issue include several groups. Most established laser- and gold nanoparticle- therapy employs the photothermal effect and delivers cell level hyperthermia [9-11]. This drug-free approach employs continuous lasers and gold colloids or specifically engineered near-infrared gold nanoparticles such as nanorods, nanoshells and others. The limitation of such an approach is in low cell specificity due to the unavoidable non-specific uptake of nanoparticles by healthy cells and due to thermal diffusion during relatively long exposure times. Another drug-free approach employs very short laser pulses to prevent thermal diffusion and instead of heat employs the purely mechanical impact of the nanoparticle-generated expanding and collapsing vapor nanobubbles (plasmonic nanobubbles) $[13,14]$. The threshold nature of plasmonic nanobubbles allowed an improvement in their cellular specificity compared to nanoparticle therapies $[8,13,14]$. The next group of therapeutic methods employs light-activated nanoparticles for the on-demand release of encapsulated drugs [10-12, 16, 17]. Nanoparticle-generated heat melts the membrane of a complex nanocarrier within minutes or less, and releases its therapeutic payload [16, 17]. Even faster release occurs via the plasmonic nanobubble-induced explosive mechanical disruption of the nanocarrier. In this case, instead of relatively slow diffusion, the payload is ejected from a nanocarrier in mere nanoseconds [14].

The possibility of combining several agents with different functions in one nanocarrier created a new class of theranostic nanoparticles [9-10, 16, 17]. For example, liposome or microcapsule may carry an encapsulated drug and attached antibodies for targeted therapy, gold nanoparticles for heating and fluorescent molecules or quantum dots for optical imaging or magnetic nanoparticles for magnetic resonance imaging. This all-in-one concept is intriguing, although the complexity of such theranostic nanoparticles will have to deal with the challenges of toxicity and stability.

Most of the papers presented in this issue consider cancer as the main target. The aggressive and resistant nature of cancer cells often leads to the failure of standard practices. This is where the new approaches discussed below can be considered as adjuvant therapies for improving patients' survival rates and quality of life as well as for making the treatment both shorter and less stressful.

\section{References}

1. [Internet] http://micro.magnet.fsu.edu/primer/museum/janssen.html.

2. Sliney DH, Trokel SL. Medical Lasers and Their Safe Use. New York, NY: Springer Verlag. 1993; ISBN: 978-1-4613-9254-5.

3. Mie G. Beiträge zur Optik trüber Medien, speziell kolloidaler Metallösungen. Annalen der Physik Vierte Folge Band. 1908;25:377-445.

4. Faraday M. The Bakerian lecture: Experimental relations of gold (and other metals) to light. Philos Trans R Soc London. 1857;147:145-81.

5. Merchant B. Gold, the noble metal and the paradoxes of its toxicology. Biologicals. 1998;26:49-59.

6. Weissleder R. A clearer vision for in vivo imaging. Nat Biotechnol. 2001;19:316-7.

7. Ambrosch P. The role of laser microsurgery in the treatment of laryngeal cancer. Curr Opin Otolaryngol Head Neck Surg. 2007;15:82-8.

8. Danysh BP, Constantinou PE, Lukianova-Hleb EY, Lapotko DO, Carson DD. The MUC1 ectodomain: a novel and efficient target for gold nanoparticle clustering and vapor nanobubble generation. Theranostics. 2012;2:777-87.

9. Menon JU, Jadeja P, Tambe P, Vu K, Yuan B, Nguyen KT. Nanomaterials for photo-based diagnostic and therapeutic applications. Theranostics. 2013; 3(3):152-166. DOI: 10.7150/thno.5327. 
10. Khlebtsov N, Bogatyrev V, Dykman L, Khlebtsov B, Staroverov S, Shirokov A, Larisa Matora L, Khanadeev V, Pylaev T, Tsyganova N, Terentyuk G. Analytical and theranostic applications of gold nanoparticles and multifunctional nanocomposites. Theranostics. 2013; 3(3): 167-180. doi:10.7150/thno.5716.

11. Zhang Z, Wang J, Chen C. Gold nanorods based platforms for light-mediated theranostics. Theranostics 2013; 3(3): 223-238. doi: $10.7150 /$ thno. 5409 .

12. Liu L, Hu R, Roy I, Lin G, Ye L, Reynolds JL, Liu J, Liu J, Schwartz SA, Zhang $X$, Yong K-T. Synthesis of luminescent near-infrared agins2 nanocrystals as optical probes for in vivo applications. Theranostics. 2013;3:109-15.

13. Lukianova-Hleb EY, Oginsky AO, Samaniego AP, Shenefelt DL, Wagner DS, Hafner JH, Farach-Carson MC, Lapotko DO. Tunable plasmonic nanoprobes for theranostics of prostate cancer. Theranostics. 2011;1:3-17.

14. Lukianova-Hleb EY, Ren X, Townley D, Wu X, Kupferman ME, Lapotko DO. Plasmonic nanobubbles rapidly detect and destroy drug-resistant tumors. Theranostics. 2012;2:976-87.

15. Wagner DS, Delk NA, Lukianova-Hleb EY, Hafner JH, Farach-Carson MC, Lapotko DO. The in vivo performance of plasmonic nanobubbles as cell theranostic agents in zebrafish hosting prostate cancer xenografts. Biomaterials. 2010;31:7567-74.

16. Leung SJ, Romanowski M. Light-activated content release from liposomes. Theranostics. 2012;2:1020-36.

17. Xiong R, Soenen S, Braeckmansa K, Skirtach AG. Toward theranostic applications of light-responsive multicompartment microcapsules. Theranostics. 2013; 3(3): 141-151. doi:10.7150/thno.5846. 